\title{
On the nature of the $\delta$ Scuti star star HD 115520
}

\author{
L. Fox Machado ${ }^{1}$, J. H. Peña ${ }^{2}$, G. Muñoz ${ }^{3}$ and B. Vargas ${ }^{3}$ \\ ${ }^{1}$ Observatorio Astronómico Nacional, Instituto de Astronomía, Universidad Nacional \\ Autónoma de México, Ensenada B.C., Apdo. Postal 877, México \\ 2 Instituto de Astronomía, Universidad Nacional Autónoma de México, México D.F., \\ Apdo. Postal 70-264, México \\ ${ }^{3}$ Escuela Superior de Ingeniera Mecánica y Eléctrica, Instituto Politécnico Nacional, \\ Av. IPN s/n, 07738 México, D.F., México
}

\begin{abstract}
As a continuation of the study of the newly found $\delta$ Scuti star HD 115520, we present a period analysis of recently acquired photometric data covering four nights, as well as some conclusions on the nature of this star.
\end{abstract}

\section{Introduction}

In 2007 Peña et al. (2007, Paper I) confirmed the belonging of HD 115520 to the $\delta$ Scuti class which was considered as a standard star in a 2005 observing run. From the relatively large scatter shown, Peña et al.(2006) consider it as a variable candidate. With this in mind, new data were acquired in two new nights in 2006 which established it as a $\delta$ Scuti star. In the present paper we present new observations which were performed in 2007 with the same instrumentation over a period of four nights and which have served to determine its periodic content. The found frequencies explain the behavior of both seasons separated by more than one year.

\section{Observations}

These were taken at the Observatorio Astrónomico Nacional, México using the $1.5 \mathrm{~m}$ telescope to which a spectrophotometer was attached. The observing season was carried out on four consecutive nights in March and April, 2007. The following observing routine was employed: a multiple series of integrations 
Table 1: Characteristics of the observed stars. The spectral types were taken from the SIMBAD database.

\begin{tabular}{lcccccc}
\hline \hline$I D$ & $\mathrm{~V}$ & $(b-y)$ & $m_{1}$ & $c_{1}$ & $\mathrm{~N}$ & SpTyp \\
\hline HD 115520 & 8.435 & 0.132 & 0.171 & 0.806 & 459 & F0 \\
HD 116879 & 7.953 & 0.272 & 0.144 & 0.634 & 120 & F5 \\
HD 114311 & 9.037 & 0.334 & 0.157 & 0.474 & 122 & F6V \\
\hline
\end{tabular}

was carried out, consisting of five $10 \mathrm{~s}$ integrations of the star to which one 10 s integration of the sky was subtracted. Two reference stars were also observed C1: HD116879 and C2: HD114311. These were observed in the following sequence to optimize the time coverage of the variable: $\mathrm{V}$, sky, $\mathrm{C} 1, \mathrm{~V}, \mathrm{~V}$, $\mathrm{C} 2, \mathrm{~V}$. A series of standard stars was also observed at the beginning and at the end of each night to transform the data into the standard system. The absolute photometric values of the 2007 campaign are provided in an archive. The accuracy of the season is deduced from the differences between the reduced and the previously reported values of the standard stars. Due to the fact that the last night was of lower quality, and hence less accurate, the mean values of the differences are calculated only from the standards of the first three nights. They are: $0.015,0.008,0.007,0.011 \mathrm{mag}$ for $\mathrm{V},(b-y), m_{1}$, and $c_{1}$, respectively.

However, since the amplitude of the star is typical of a $\delta$ Scuti star $(\sim$ $20 \mathrm{mmag}$, see Figure 4), we preferred to analyze the data for the periodic content through differential photometry in the $y$ filter for which use was made of the reference stars $\mathrm{C} 1$ and $\mathrm{C} 2$ to increase the accuracy of the photometry to thousands of magnitude. Table 1 lists the characteristics of the observed stars. A magnitude value of the reference stars was interpolated at the time of the variable and the final values, to which the average value of each night was subtracted, are presented in Table 1 . The whole reduction procedure is shown in Figure 1 for the night of March 30/31. The 2006 season was reduced in the same fashion to match the newly acquired data.

\section{Frequency determination}

With the relatively few data points acquired in the 2006 season (only two short nights) we were able to demonstrate the star's variability and found evidence of at least two close frequencies which might explain the resulting beating behavior of the light curve. Since the new photometric data is constituted of four long consecutive nights, we are now able to determine the pulsational frequencies with greater precision. Two numerical packages were utilized: Period04 (Lenz and Breger, 2004) and ISWF (Alvarez et al., 1998). With Period04 the first run examined gave a frequency of $17.8643 \mathrm{c} / \mathrm{d}$ with an amplitude of 0.0140 




Figure 1: $Y$ variation of the observed stars HD 115520, C1 and C2 on the night of March $30 / 31,2007$. $Y$ axis is $V$ in magnitudes, $X$ axis is time (time shown=HJD 2454100.0)

mag in the frequency interval between 0 and $30 \mathrm{c} / \mathrm{d}$ with a step rate of 0.0150 . Prewhitening of this frequency consecutively yielded the results shown in Figure 2. On the other hand, the ISWF package yielded the following frequencies (in c/d) listed in diminishing amplitudes (in parentheses, in mmag) 17.850 (13.877); 14.7786 (10.334); 17.4527 (6.415); 13.5217 (4.236) and 18.1831 (3.973).

As can be seen, the two previously determined main frequencies, although slightly numerically different are confirmed. In the 2006 season we obtained 18.82 and $14.63 \mathrm{c} / \mathrm{d}$. Given the complex window function of observations on only two nights from only one observatory, we might consider them the same. On the other hand, when the whole dataset was utilized with a step rate of 0.00015 , Period04 yielded peaks at 17.8373 and $14.7537 \mathrm{c} / \mathrm{d}$, (see Figure 3 and Table 2). The rest of the frequencies might be disregarded because they do not significantly improve the residuals. Their peaks are indistinguishable from 


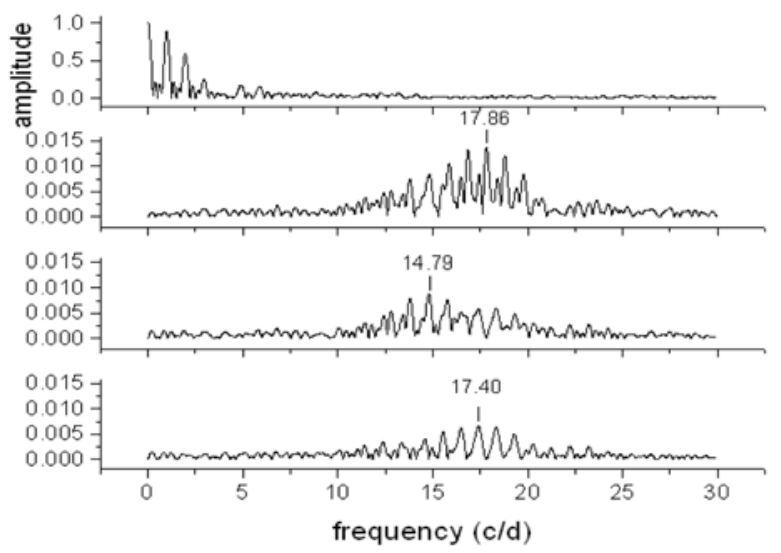

Figure 2: Periodograms of the four consecutively observed nights in 2007. From top to bottom, window, first frequency obtained at $17.86 \mathrm{c} / \mathrm{d}$, periodogram after prewhitening this with a resulting peak at $14.79 \mathrm{c} / \mathrm{d}$, and finally the prewhitened histogram of the two previously determined frequencies with a peak at $17.400 \mathrm{c} / \mathrm{d}$.

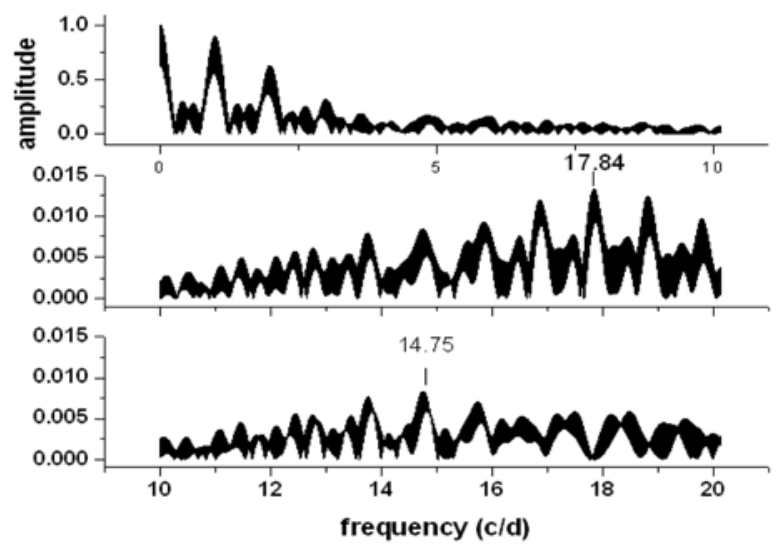

Figure 3: Periodograms of all the observed nights. From top to bottom, window, first frequency obtained at $17.8375 \mathrm{c} / \mathrm{d}$, periodogram after prewhitening this with a resulting peak at $14.7537 \mathrm{c} / \mathrm{d}$, and finally the prewhitened histogram of the two previously determined frequencies with a peak at $16.5121 \mathrm{c} / \mathrm{d}$. 
Table 2: Frequencies, amplitudes and phases derived

\begin{tabular}{llll}
\hline \hline & Frequency (c/d) & Amplitude (mag) & Phase \\
\hline F1 & 17.8375 & 0.0131 & 0.1028 \\
F2 & 14.7537 & 0.0108 & 0.2612 \\
F3 & 16.5121 & 0.0070 & 0.5646 \\
\hline
\end{tabular}


Figure 4: $y$ variation of HD 115520 (dots). $\mathrm{Y}$ axis is $\mathrm{y}$ in magnitudes, $\mathrm{X}$ axis is time.

each other due to the aliasing caused by the window function. Therefore, we will consider as definitive only the first two frequencies listed in Table 2. Figure 4 shows the light curves of the six observed nights.

\section{Physical parameters}

As it has been already described in paper I, we carried out a well-known procedure to determine reddening as well as unreddened colors using the photometric mean $u v b y-\beta$ values reported in Table 3 . Table 4 lists the reddening, the unreddened indexes, the absolute magnitude, and the distance. Its position on the $\left[m_{1}\right]-\left[c_{1}\right]$ diagram established it to be an A8V star. Its temperature and 
Table 3: Mean values of the uvby photometry (in mag) of HD 115520 from the two seasons

\begin{tabular}{llll}
\hline \hline & average & sigma & $\mathrm{N}$ \\
\hline $\mathrm{V}$ & 8.4305 & 0.0178 & 579 \\
$(b-y)$ & 0.1334 & 0.0070 & 584 \\
$m_{1}$ & 0.1701 & 0.0051 & 580 \\
$c_{1}$ & 0.8068 & 0.0139 & 584 \\
$\beta$ & 2.8108 & 0.0133 & 67 \\
\hline
\end{tabular}

log of surface gravity can be determined by locating HD 115520 in the $(b-y)_{0}$ vs. $c_{0}$ grids of Lester et al. (1986, hereafter LGK86) (Figure 5); the values we determine are $7700 \mathrm{~K}$ and 4, respectively. As was stated in Paper I, we compared our results with those in a paper by Behr (2003) who found an effective temperature $T_{\text {eff }}$ of $8199(+449,-317)$, a log g $4.63(+0.34,-0.23)$, an $[\mathrm{Fe} / \mathrm{H}]$ $0.62(+-0.13)$ and a stellar type belonging to the main-sequence for this star. Although Behr (2003) has evaluated physical parameters for this star, and his numerical values coincide with ours, we feel that we have more data to determine the physical characteristics. Nevertheless, we have employed his reported metallicity of HD 115520 to discriminate between the models that explain the star's behavior.

\section{The evolutionary status of HD 115520}

The determination of the evolutionary stage of a field star requires precise estimates of its global parameters. In the case of HD 115520 the distance as determined from Strömgren photometry is $140 \mathrm{pc}$ which leads an $M_{V}$ of 2.86 mag by using the calibrations of Shobbrook (1984). On the other hand, the distance value of 300 pc estimated from a parallax of $3.29 \pm 0.97$ mas provided by the Hipparcos catalogue (Perryman et al. 1997) yields an $M_{V}$ of 1.02 mag which is quite different from the photometric value. This ambiguity can be explained by the uncertainties in the determination of each measured distance. The large relative error $(\sigma(\pi)) / \pi \sim 0.30)$ of the Hipparcos parallax for HD 115520 implies an $\sigma\left(M_{V}\right)>0.5 \mathrm{mag}$, whereas in the present paper the uncertainty in the apparent magnitude derived as explained in Peña \& Sareyan (2006) from the standard deviation of 579 data points of the two seasons gives an $m_{V}=8.4305 \pm 0.0178$ (see Table 3) and an $\sigma\left(M_{V}\right)<0.1 \mathrm{mag}$. Although this latter value does not include the uncertainty in $M_{V}$ due to the photometric calibrations which can be as large as $0.3 \mathrm{mag}$ for early type stars (e.g. Balona \& Shobbrook 1984), we think that the photometric distance is more reliable than the trigonometric one because different photometric calibrations (Balona 
Table 4: Reddening and unreddened parameters of HD 115520

\begin{tabular}{lccccccc}
\hline \hline$E(b-y)$ & $(b-y)_{0}$ & $m_{0}$ & $c_{0}$ & $V_{0}$ & $M_{v}$ & $D M$ & dst $(\mathrm{pc})$ \\
\hline 0.000 & 0.135 & 0.170 & 0.807 & 8.43 & 2.68 & 5.75 & 141 \\
\hline
\end{tabular}

\& Shobbrook (1984) and Nissen (1988)) lead to similar distance values for HD 115520. Furthermore, similar values of $m_{V}$ for HD 115520 have already been reported in previous papers (Olsen 1983, Crawford \& Perry 1989, Paper I). Therefore, we will use the photometrically determined distance to tray to establish the evolutionary status of HD 115520.

Figure 6 shows the observed position of HD 115520 (asterisk) in the HR diagram and its associated uncertainty (cross upon the asterisk). PMS and post-MS evolutionary tracks giving a range of masses between $1.45-1.60 M_{\odot}$ for HD 115520 are shown with dotted and continuous lines respectively. These evolutionary sequences were computed by using the CESAM evolution code (Morel 1997) with an input physics appropriate to $\delta$ Scuti stars and a chemical initial composition of $Z=0.013$ and $Y=0.28$. Also shown are the theoretical pre-MS instability strip boundaries of the first three radial modes obtained by Marconi \& Palla (1998).

According to the models depicted in Fig. 6 HD 115520 could either be in pre-MS stage with an age between 15-20 Myr or post-MS stag with an age between 500-700 Myr. In the former case, the age was estimated as the time spent by the star travelling from the birthline to the ZAMS in the HR diagram according to the isochrones given by Tout et al. (1999).

As shown by Suran et al. (2001) non-radial oscillation spectra in the low frequency domain can be used to discriminate between the pre- and post-MS stage. In the present case, however, this is seldom possible since the two detected peaks in HD 115520 are most likely due to radial oscillations. In fact, we have tried to reproduce the observed periods computing linear adiabatic pulsation models of HD 115520 for some selected pre- and post-MS models located within the error box in Figure 6, but no satisfactory fit between observed and theoretical frequencies was found. Therefore, more observational efforts are required to establish the true nature of this interesting object.

\section{Conclusions}

We have presented the analysis of new uvby photometric observations of $\delta$ Scuti star HD 115520 carried out during four nights in March and April, 2007 at the Observatorio Astronómico Nacional, México. These data were added to the previously observed two nights in 2006 resulting a total of 580 data 


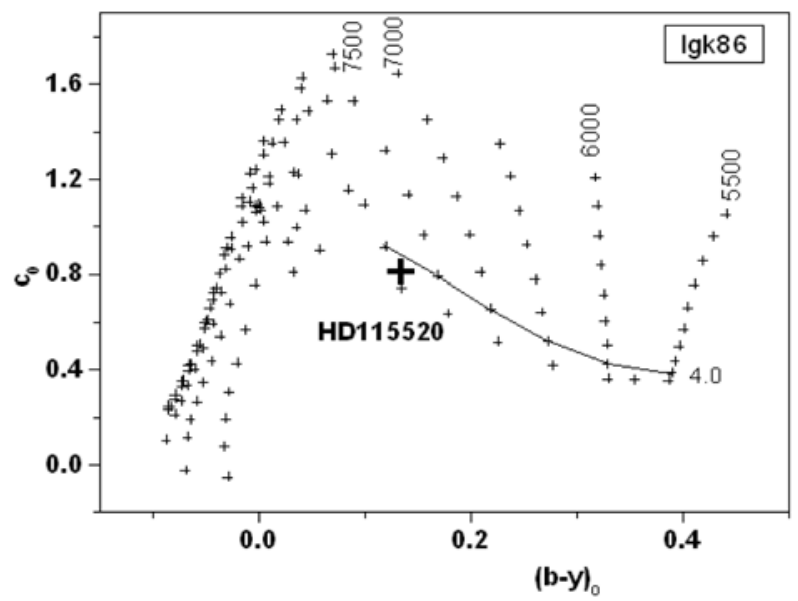

Figure 5: Location of the photometric data of HD 115520 in the grids of LGK86.

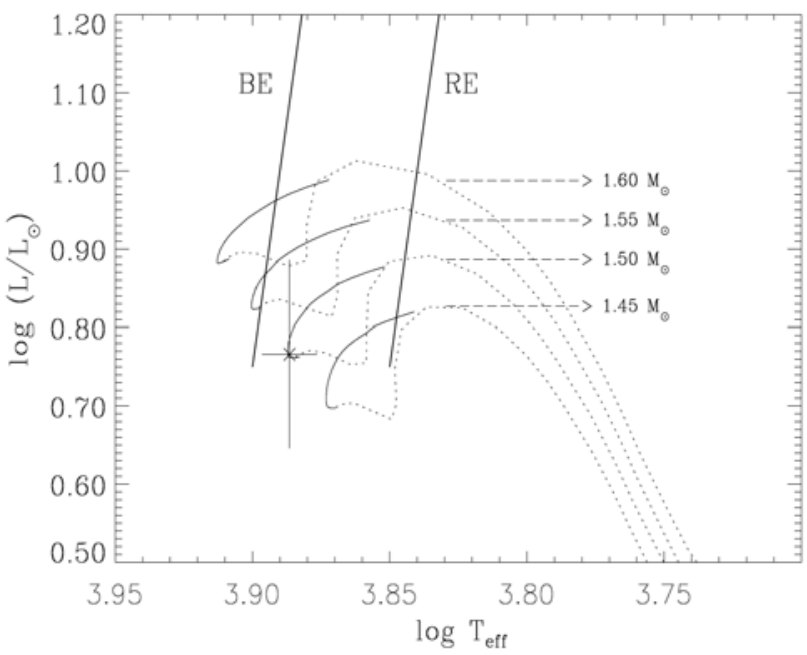

Figure 6: Position of HD 115520 in the HR diagram. 
points of uvby photometry which allowed us to search for the true nature of this $\delta$ Scuti variable. The two oscillations frequencies detected in 2006 have been confirmed in this season. We have found that both stages pre-MS and post-MS are possible to account for the observed luminosity and temperature of the star. We thus conclude that HD 115520 represents an good candidate for asteroseismological studies of young $\delta$ Scuti stars.

Acknowledgments. We would like thank the assistance of the staff of the OAN during the observations. This paper was partially supported by Papiit IN108106. GM and BV thank the OAN for allowing the use of the telescope time and to Dr. Jorge Sosa of IPN for the support. J. Miller and J. Orta did the proofreading and typing, respectively. This article has made use of the SIMBAD database operated at CDS, Strasbourg, France and ADS, NASA Astrophysics Data Systems hosted by Harvard-Smithsonian Center for Astrophysics.

\section{References}

Alvarez, M., Hernandez, M. M., Michel, E., et al. 1998, A\&A 340, 149

Balona, L. A., \& Shobbrook, R. R. 1984, MNRAS 211, 375

Behr, B. B. 2003, ApJS 149, 101

Crawford, D. L., \& Perry, C. L. 1989, PASP 101, 601

Lenz, P., \& Breger, M. 2005, CoAst 146, 53

Lester, J. B., Gray, R. O., \& Kurucz, R. I. 1986, ApJ 61, 509

Marconi, M., \& Palla, F. 1998, ApJ 507, 141

Morel, P. 1997, A\&AS 124, 597

Nissen, P. 1988, A\&A 199, 146

Peña, J. H., \& Sareyan, J. P. 2006, RevMexAA 42, 179

Peña, J. H., Sareyan, J. P., Cervantes Sodi, B., et al. 2007, RevMexAA 43, 217

Olsen, E. H. 1983, A\&AS 54, 55

Perryman, M. A. C., Lindegren, L., Kovalevsky, J., et al. 1997, A\&A 323, 49

Shobbrook, R. R. 1984, MNRAS 211, 659 